\title{
Male X-chromosome Mosaicism leading to Carrier Phenotype and inheritance of Chronic Granulomatous Disease
}

Stephanie Harris MD ${ }^{1}$, Helen Braggins ${ }^{2}$, Karin van Leeuwen $\mathrm{MSc}^{3}$, Kimberly Gilmour $\mathrm{PhD}^{2}$, Matthew S Buckland MD PhD ${ }^{2,4,5}$, Dirk Roos $\mathrm{PhD}^{3}$, David M Lowe MD PhD ${ }^{4,5}$

${ }^{1}$ Department of Infectious Diseases, Royal Free London NHS Foundation Trust, London, UK

${ }^{2}$ Department of Immunology, Great Ormond Street Hospital, London, UK

${ }^{3}$ Sanquin Research, Amsterdam, the Netherlands

${ }^{4}$ Institute of Immunity and Transplantation, University College London, London, UK

${ }^{5}$ Department of Immunology, Royal Free London NHS Foundation Trust, London, UK

Corresponding author: $\quad$ Dr David Lowe

Institute of Immunity and Transplantation

University College London

Royal Free Campus

Pond Street

London

NW3 2QG

United Kingdom

d.lowe@ucl.ac.uk

+44 (0)207 7940500

Funding: Nil

Word count: 946 words

Clinical implications: We present the first description of a male with X-chromosome mosaicism who has clinical features of the X-linked CGD carrier state and passed a $C Y B B$ mutation to a daughter and subsequently affected grandson: clinicians should be aware of this possibility.

Key-words: Chronic Granulomatous Disease; CGD; mosaicism

Conflicts of Interest: The authors declare no conflicts of interest. 
1 To the Editor,

3 Chronic Granulomatous Disease (CGD) is a genetic primary immunodeficiency caused by mutations in the nicotinamide adenine dinucleotide phosphate (NADPH) oxidase enzyme complex. This leads to impaired production of reactive oxygen species and impaired killing of ingested bacteria and fungi. About two thirds of cases are in males with X-linked CGD caused by mutations in the $C Y B B$ gene, coding for gp91 ${ }^{\text {phox }}$, found at Xp21.1-p11.4 [1]; in approximately $10-12 \%$ of families these $C Y B B$ mutations are believed to arise de novo.

9 Female carriers of X-linked CGD exhibit a range of phenotypes, although in one series $37 \%$ were completely asymptomatic and 52\% had no features of CGD disease [2]. Common manifestations in those who are symptomatic include recurrent oral ulceration, photosensitive rashes and discoid lupus erythematosus (DLE) [2-4], the latter seen in between 9\% [2] and $40 \%$ [3] depending on population. They can also be affected by gastrointestinal conditions similar to classic inflammatory bowel disease (approximately $4 \%[2,3])$ and have an increased predisposition to other lupus-like autoimmune manifestations. Female carriers can also present with recurrent or suppurative infections $(15 \%$ had severe infections in a recent report [2]) and indeed with extremely skewed lyonisation they can essentially develop the full CGD syndrome [1]. Thus the carriage state can also confer a significant burden of disease.

We present a patient who was identified after his grandson was diagnosed with X-linked CGD. The grandson, who is the index case, was noted to have abnormal neutrophil functional tests and a c.1253C>T (p.Ser418Phe) $C Y B B$ mutation, and has subsequently undergone haematopoietic stem cell transplantation. During family screening his mother was found to be a carrier of this variant, but not his maternal grandmother. It was noted that instead his maternal grandfather had some features consistent with a carrier state. This person (the 
maternal grandfather of the index case) is 51 years old with a history of asthma, epilepsy, hypertension and a significant infection history with two previous episodes of pneumonia or "pleurisy" and two episodes of cellulitis. He reported sun sensitivity, arthralgia and frequent 'blistering' of the lips. He had previously been referred to dermatology with a rash on his upper chest that was diagnosed on biopsy as Jessner's lymphocytic infiltrate of the skin (JLIS) (Figure 1A \& 1B) treated successfully with hydroxychloroquine. JLIS demonstrates very similar characteristics clinically and on histological examination to discoid lupus erythematosus [5]. He reported recurrent lower respiratory tract infection requiring antibiotics as a child and a requirement for antibiotics twice a year even in adulthood. A CT chest showed mild left lower lobe bronchiectasis (Figure 1C).

This maternal grandfather of the index case was found to have an abnormal Nitro-blue tetrazolium (NBT) test and dihydrorhodamine (DHR) oxidation, both of which demonstrated two populations of neutrophils upon stimulation, one capable of respiratory burst and one with an impaired burst (Figure 2A \& 2B). This was confirmed on repeated testing. Gp91 $1^{\text {phox }}$ expression in these cells showed one positive and one negative peak (Figure 2C). Genetic analysis from blood and a salivary sample via Ion Torrent ${ }^{\mathrm{TM}}$ next generation sequencing confirmed that he in fact has three $\mathrm{X}$-chromosome variants, two of which carry a novel $C Y B B$ mutation at position c.1253 (c.1253C > T [p.Ser418Phe] in 44\% of reads, c.1253C >G [p.Ser418Cys] in 7-8\% of reads and the remainder wild-type; Supplementary Figure 1). He had undergone vasectomy and thus could not provide a semen sample for further analysis. The brother and the three other daughters of the maternal grandfather of the index case underwent genetic screening but all were negative for these mutations (Figure 2D).

\section{Discussion}


It has previously been reported that male patients with X-linked CGD can have small populations of normal cells [6], suggesting mutation in early embryogenesis, or early reversion in some cell lines after the mutation arose in the oocytes. There has also been a case report of an X-linked CGD patient's brother with Klinefelter's syndrome having carrier status [7], due to inheritance of both the mutated allele of $C Y B B$ and the normal allele. Case reports of triple mosaic female carriers who had populations of neutrophils with two different deletion mutations in $C Y B B$ and the normal variant have been described [8], as has a lateonset somatic mutation in $C Y B B$ with subsequent mosaicism in haematopoietic cells in a female [9]. However, this is the first report to our knowledge of a fertile male acting as a true 'carrier' for X-linked CGD with a compatible clinical syndrome (the 'JLIS' is likely to represent discoid lupus as seen in the CGD carrier state) and inheritance of the diseasecausing variant in offspring. Both wild-type and mutant forms were present in blood and saliva: this raises the possibility of either early reversion of a germline mutation during embryogenesis or a mutation arising during an early multicellular stage with both populations of cells increasing proportionally. Given that there is no evidence that his brother has the mutation or clinically that there are other carriers in the family before him we conclude it is likely that this is a de novo mutation.

Male carriage of X-linked conditions in this manner would not be picked up via routine genetic screening of affected families and can lead to unintentional inheritance of the mutation without appropriate genetic counselling. Indeed in this case the maternal grandfather passed the gene on to one of his four daughters who subsequently had an affected child. There is a potential for grandfathers who are mosaics for X-linked conditions to pass on the genetic mutation to future children and, in diseases such as CGD, carriers themselves 
74 are aware of this possibility and consider at least functional testing of maternal grandfathers 75 in the presence of compatible symptoms. 


\section{References}

1. Roos D, Kuhns DB, Maddalena A, Roesler J, Lopez JA, Ariga T, et al. Hematologically important mutations: X-linked chronic granulomatous disease (third update). Blood Cells Mol Dis 2010,45:246-265.

2. Marciano BE, Zerbe CS, Falcone EL, Ding L, DeRavin SS, Daub J, et al. X-linked carriers of chronic granulomatous disease: Illness, lyonization, and stability. J Allergy Clin Immunol 2018,141:365-371.

3. Battersby AC, Braggins H, Pearce MS, Cale CM, Burns SO, Hackett S, et al. Inflammatory and autoimmune manifestations in X-linked carriers of chronic granulomatous disease in the United Kingdom. J Allergy Clin Immunol 2017,140:628630

4. Battersby AC, Cale AM, Goldblatt D, Gennery AR. Clinical manifestations of disease in X-linked carriers of chronic granulomatous disease. J Clin Immunol 2013,33:12761284.

5. Lipsker D, Mitschler A, Grosshans E, Cribier B. Could Jessner's lymphocytic infiltrate of the skin be a dermal variant of lupus erythematosus? An analysis of 210 cases. Dermatology 2006,213:15-22.

6. Yamada M, Okura Y, Suzuki Y, Fukumura S, Miyazaki T, Ikeda H, et al. Somatic mosaicism in two unrelated patients with $\mathrm{X}$-linked chronic granulomatous disease characterized by the presence of a small population of normal cells. Gene 2012,497:110-115.

7. Gill HK, Kumar HC, Cheng CK, Ming CC, Nallusamy R, Yusoff NM, et al. X-linked chronic granulomatous disease in a male child with an X-CGD carrier, Klinefelter brother. Asian Pac J Allergy Immunol 2013,31:167-172.

8. de Boer M, Bakker E, Van Lierde S, Roos D. Somatic triple mosaicism in a carrier of X-linked chronic granulomatous disease. Blood 1998,91:252-257.

9. Wolach B, Scharf Y, Gavrieli R, de Boer M, Roos D. Unusual late presentation of Xlinked chronic granulomatous disease in an adult female with a somatic mosaic for a novel mutation in CYBB. Blood 2005;105:61-6. 


\section{Figure Legends}

Figure 1. A. Photograph of the rash diagnosed as Jessner's lymphocytic infiltrate (diameter approximately $2 \mathrm{~cm}$ ). B. Histology from a skin lesion demonstrating superficial and deep dermal perivascular inflammation, normal epidermis on low power (left) and detail of the predominantly lymphocytic infiltrate on higher power (right). C. Axial section from CT thorax showing dilated bronchi larger than accompanying blood vessels, consistent with mild bronchiectasis (arrows).

Figure 2. A. NBT test (see Supplementary methods). The maternal grandfather of the index case ('patient', right) demonstrates two populations of cells, one able and one unable to reduce NBT and form blue formazan. B. Dihydrorhodamine test (see Supplementary methods). Rhodamine-1,2,3-FITC results are shown for a control (left) and the maternal grandfather of the index case ('patient', right) without stimulation (red histogram) and after stimulation with PMA (blue histogram), again indicating two cell populations in the maternal grandfather. C. gp91 $1^{\text {phox }}$ expression (see Supplementary methods) in neutrophils; isotype control (red histogram) and gp91phox stained (blue histogram) results are shown for a control (left) and the maternal grandfather of the index case ('patient', right) who has a population of gp91 ${ }^{\text {phox }}$-negative cells. D. Family pedigree based on Ion Torrent ${ }^{\mathrm{TM}}$ next generation sequencing, indicating transmission of presumed deleterious $C Y B B$ mutation from the maternal grandfather to one daughter and then to a grand-son (index case) who presented with X-linked CGD. 
Figure 1

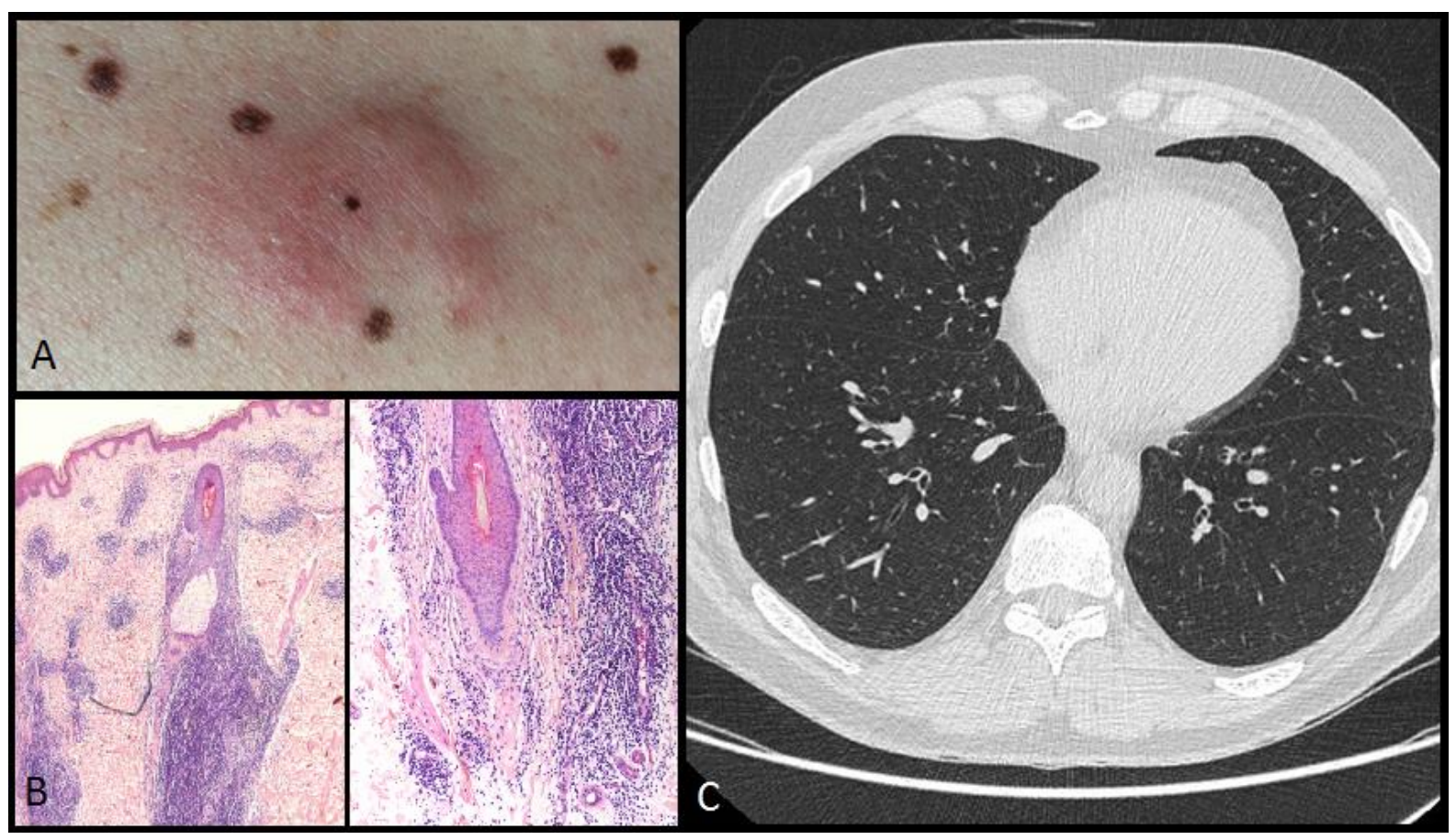


Figure 2

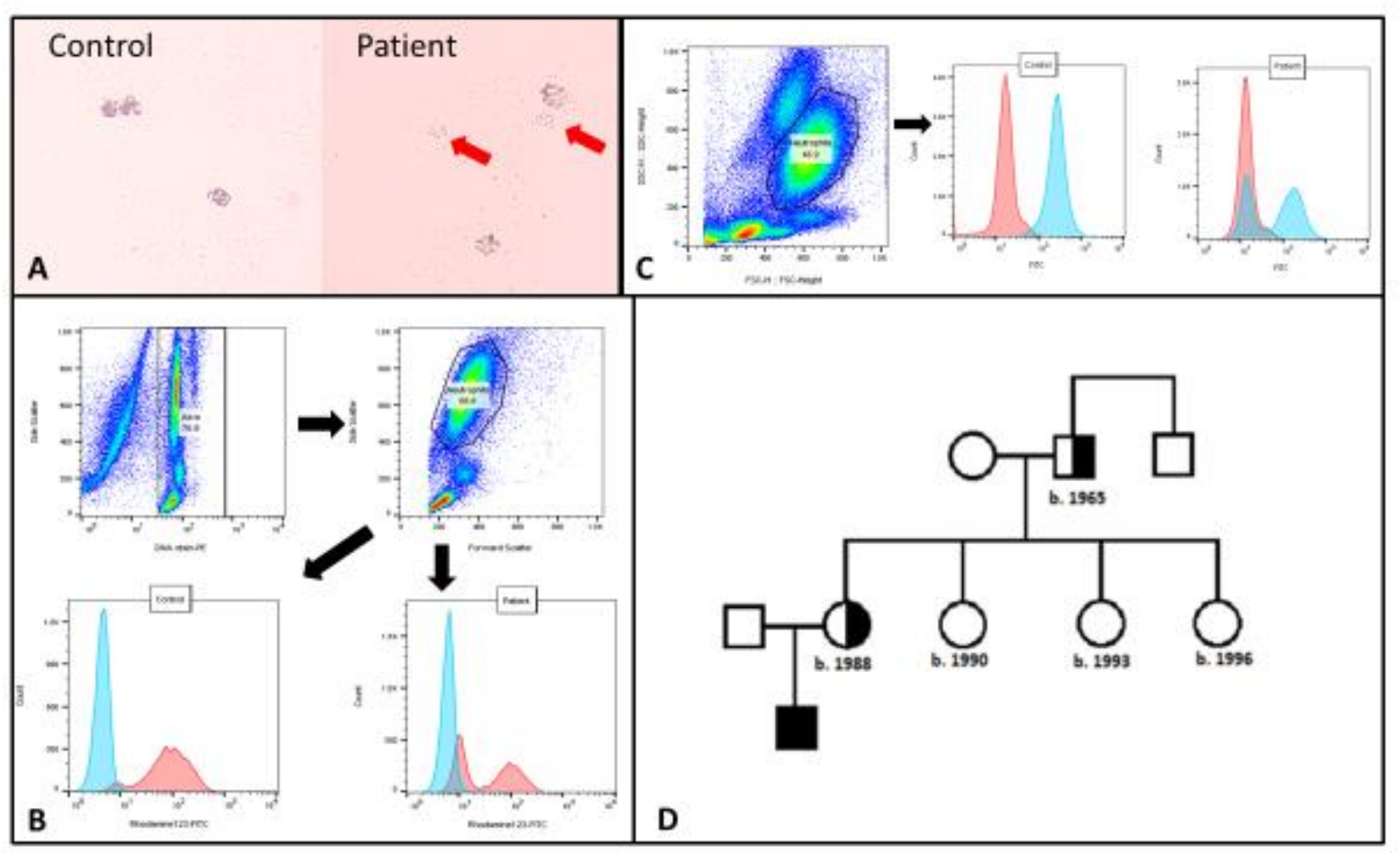




\section{Supplementary Information}

\section{Supplementary Figure 1}

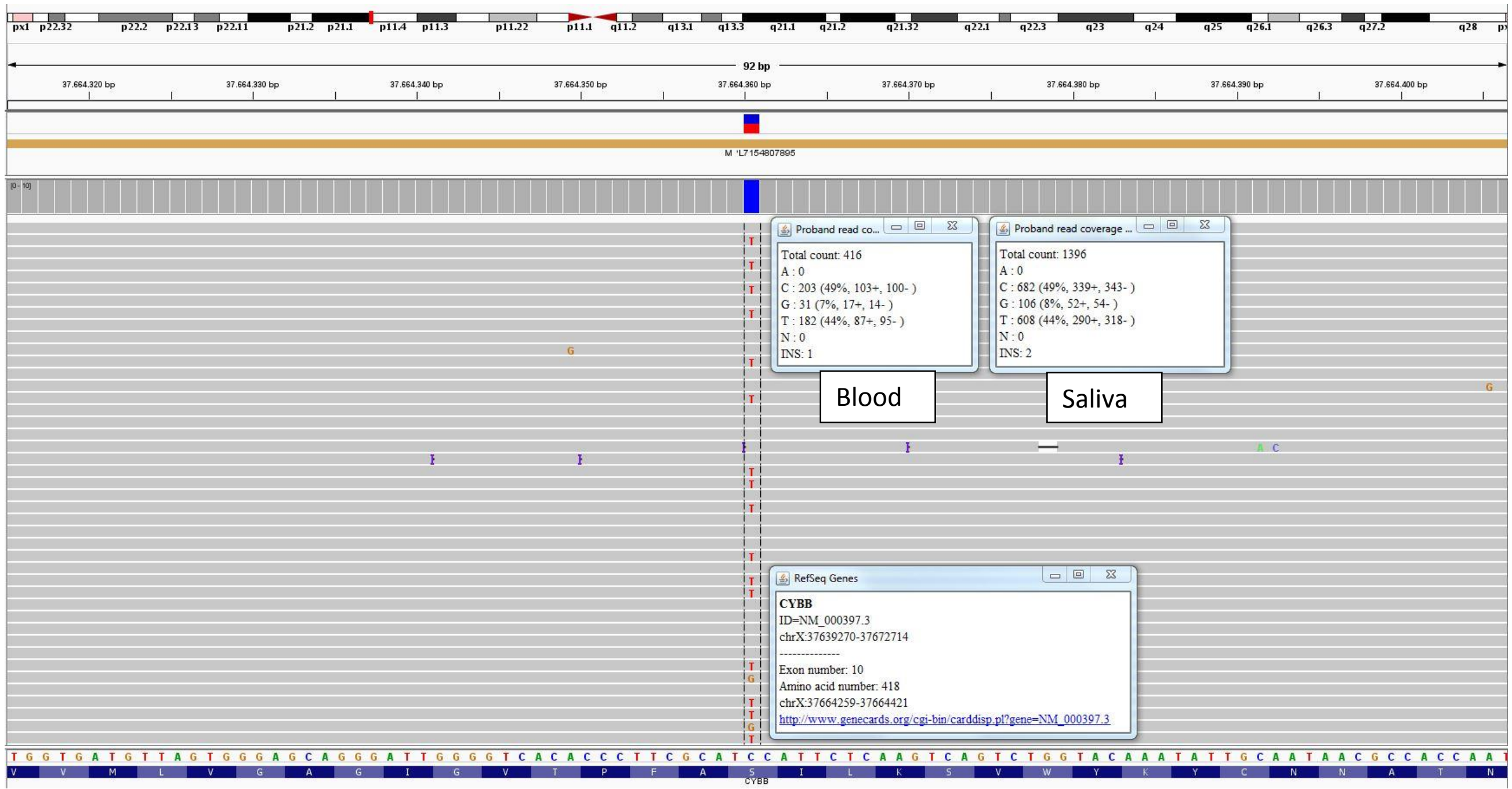




\section{Supplementary Methods}

NBT test. This was performed in accordance with published reports [1]. Briefly, $100 \mu 1$ of EDTA whole blood was incubated with $100 \mu 1$ of $0.2 \%$ NBT (Sigma) and $100 \mu \mathrm{lof} 10 \mu \mathrm{g} / \mathrm{ml}$ phorbol 12-myristate 13-acetate (PMA) (Sigma) for 25 minutes at $37^{\circ} \mathrm{C}$, then 25 minutes at room temperature before mixing with $500 \mu \mathrm{l}$ of $2 \%$ acetic acid and trace crystal violet; $10-\mu \mathrm{l}$ aliquots were added to counting slides and examined at x40 magnification.

DHR test. This was performed in accordance with published reports [2]. The PhagoBurst ${ }^{\mathrm{TM}}$ kit (Glycotope Biotechnology) was used on heparinised whole blood according to manufacturers' instructions and samples processed on a FACSCalibur flow cytometer. For analysis a DNA stain was used to identify live cells and then neutrophils were gated by forward and side scatter (Figure 2).

Gp91 ${ }^{\text {phox }}$ expression. This was determined in neutrophils (gated by forward and side scatter; Figure 2) via flow cytometry using 7D5 mAb against gp91 ${ }^{\text {phox }}$ (IgG1, LifeSpan BioSciences) and a FITC-conjugated rabbit-anti-mouse IgG.

\section{References}

1. Meerhof LJ, Roos D. Heterogeneity in chronic granulomatous disease detected with an improved nitroblue tetrazolium slide test. J Leukoc Biol 1986,39:699-711.

2. Vowells SJ, Sekhsaria S, Malech HL, Shalit M, Fleisher TA. Flow cytometric analysis of the granulocyte respiratory burst: a comparison study of fluorescent probes. J Immunol Methods 1995,178:89-97.

\section{Supplementary Figure Legend}

Supplementary Figure 1. Results of Ion Torrent ${ }^{\mathrm{TM}}$ next generation sequencing of the CYBB gene in the patient's blood and saliva. 